\title{
Analysis of low dose level volumes in intensity modulated radiotherapy and 3-D conformal radiotherapy
}

\author{
Varadharajan Ekambaram ${ }^{1}$, Ramasubramanian Velayudham ${ }^{2}$ \\ ${ }^{I}$ Department of Radiation Oncology, Billroth Hospitals LTD, Chennai, India. \\ ${ }^{2}$ Nuclear and Medical Physics Division, VIT University, Vellore, India.
}

Received January 26, 2014; Revised March 26, 2014; Accepted March 30, 2014; Published Online May 04, 2014

\section{Original Article}

\begin{abstract}
Purpose: To analyze the low dose volume regions in four facets: Analysis 1: low dose volume regions were compared between 3-dimensional conformal radiotherapy (3DCRT) and intensity modulated radiotherapy (IMRT) plans for each case; Analysis 2: the effect on low dose volume in 3DCRT as the number of fields are increased; Analysis 3: the same effect in IMRT, and Analysis 4: above two analysis were inter-compared between the two modalities. Methods: For this work 18 patients were taken for which both 3DCRT and IMRT plans with varying number of beams were planned using Anisotropic Analytical Algorithm (AAA) in Eclipse 10 Version Treatment Planning System with two to nine beams and five to nine beams, respectively. The plans were analyzed on the basis of conformity index and dose to the critical structures. Results: In Analysis 1, 5 Gy volume region was greater for IMRT than for 3DCRT but the $10 \mathrm{~Gy}, 15 \mathrm{~Gy}$ and $20 \mathrm{~Gy}$ volume regions were smaller for IMRT plans. In Analysis 2 and 3 shows that as the number of fields increases the low dose volume regions also increases. In Analysis 4, the effect pronounced due to increase in the beam portals is similar in 3DCRT as well as IMRT. Also it was seen that with same number of beams for both IMRT and 3DCRT, low dose volume region is higher in 3DCRT than IMRT. Conclusion: Low dose volume regions are a major concern in 3DCRT and IMRT plans with multiple beams because of its risk of secondary cancer incidence. This study concludes that by increasing number of beams low dose volume regions increases in 3DCRT and IMRT. It shows that the low dose volume regions are slightly higher in IMRT than 3DCRT, however with proper optimization the volume of tissue receiving $10 \mathrm{~Gy}, 15 \mathrm{~Gy}$ and $20 \mathrm{~Gy}$ can be reduced.
\end{abstract}

Keywords: Intensity Modulated Radiotherapy; Three Dimensional Conformal Radiotherapy; Treatment Planning System; Analytical Anisotropic Algorithm; Low Dose Regions

\section{Introduction}

The discipline of Radiation Oncology is growing with continued advancements in imaging technique, treatment planning and delivery technique to achieve precise and accurate treatment. The advancements in the treatment delivery results in Three Dimensional Conformal Radiation Therapy (3DCRT), Intensity Modulated Radiation Therapy (IMRT), dynamic targeting Image Guided Radiation Therapy (IGRT) and dynamic adaptive radiation therapy in which IMRT and IGRT involves high dose gradients within few

Corresponding author: Varadharajan Ekambaram; Department of Radiation Oncology, Billroth Hospitals LTD, Chennai, India.

Cite this article as:

Ekambaram V, Velayudham R. Analysis of low dose level volumes in intensity modulated radiotherapy and 3-D conformal radiotherapy. Int J Cancer Ther Oncol 2014; 2(3):02032.

DOI: $10.14319 /$ ijcto.0203.2 mm. ${ }^{1-4}$ The major goal of radiation therapy is the delivery of prescribed dose as accurately as possible to tumor region while minimizing the dose to the neighboring normal tissues.

A good treatment planning requires the following criteria such as (a) dose distribution within the tumor volume be reasonably uniform (b) maximum dose to the tissues in beam direction should not be excessive (c) normal critical structures in the beam should not receive doses near or beyond tolerance. The above mentioned criteria can be achieved by combining two or more fields. In advanced treatment planning modalities such as 3DCRT and IMRT to get better conformity and dose homogeneity, more number of fields are used. In 3DCRT the maximum number of fields used is two to six and in IMRT the maximum number of fields used is about nine to achieve acceptable results. The 
use of multiple fields reduces large dose regions considerably but increases the low dose regions. ${ }^{5-10}$

After skin, colorectal, lung, breast \& prostate cancer, second primary cancers are the sixth most common group of malignancies in oncology. These secondary cancers also arise from radiation therapy given to primary cancer. Typically, these secondary cancers occur around the already irradiated field. Bone and soft tissue sarcomas are the most frequent second malignancies following radiation therapy, but skin, brain, thyroid, and breast cancer also can occur. The risk of secondary cancer is high when radiation therapy was given at an early age to a primary cancer or during rapid growth of a tissue. These cancers are mainly due to the low dose delivered to a larger volume of the patient. So it becomes essential to analyze these low dose volumes while planning a treatment. ${ }^{11,12}$

The aim of the work was to analyze the low dose volume regions. The project was analyzed in four different facets: 1 ) Low dose volume regions were compared between 3DCRT and IMRT plans for each case; 2) Analysis of the effect on low dose volumes in 3DCRT as the number of fields are increased; 3) Analysis of the effect on low dose volumes in IMRT as the number of fields are increased and 4) The above two analysis were compared to study the similarity or difference between the two modality.

\section{Methods and Materials}

For this work about 18 patients of different clinical diagnostic sites like head and neck, chest and pelvic regions were taken. This study included the cases of 5 hypopharynx cancer, 5 lung cancer, 4 endometrial cancer and 4 prostate cancer. All the patients were immobilized using aquaplast mask immobilization system. Patients were positioned on a wide bore Siemens computed tomography (CT) simulator flat couch with the help of lasers. Furthermore, radio opaque markers were placed during the immobilization procedure to guide the isocenter shift. For all the patients, CT scan images of slice thickness $2 \mathrm{~mm}$ were obtained. After CT simulation, DICOM images were transferred to Eclipse treatment planning system (version 10.0; Varian Medical Systems, Palo Alto, California, USA). Planning target volumes (PTV) and Organs at Risk (OAR) were delineated on the axial CT slices.

For head and neck patients, PTV, spinal cord, brainstem, left and right parotids and mandible were contoured. For lung cases, PTV, spinal cord, heart and contralateral Lung were delineated. In the case of endometrial cancer, PTV, bladder, rectum, bowels and femoral heads were contoured. In prostate cancer, gross target volume (GTV) (only prostate), CTV (GTV + $1 \mathrm{~cm}+$ seminal vesicle), PTV $(\mathrm{CTV}+1 \mathrm{~cm})$, bladder, rectum and femoral heads were contoured. For treatment planning, $6 \mathrm{MV}$ and $15 \mathrm{MV}$ X-rays from Clinac DMX (Varian Medical Systems, Palo Alto, California, USA ) integrated with 120 leaves millennium multi leaf collimator was used. For all the patients, both the 3DCRT and IMRT plans were performed in Eclipse treatment planning system of version 10 and the dose distribution was calculated using Analytical Anisotropic Algorithm (AAA) with grid size set to $1.25 \mathrm{~mm}$. All IMRT plans were done using $6 \mathrm{MV}$ X-rays while for 3DCRT plans; the energy selection was dependent on the depth. For example, for depths greater than $12 \mathrm{~cm}, 15$ MV X-rays were used. Eclipse uses helios for IMRT optimization. Eclipse version 10 is 2 to ten times faster than its previous versions. In this work, the low dose volume regions were analyzed in four different studies. Firstly 3DCRT and IMRT plans with 2 fields to 9 fields and 5 fields to 9 fields were studied respectively.

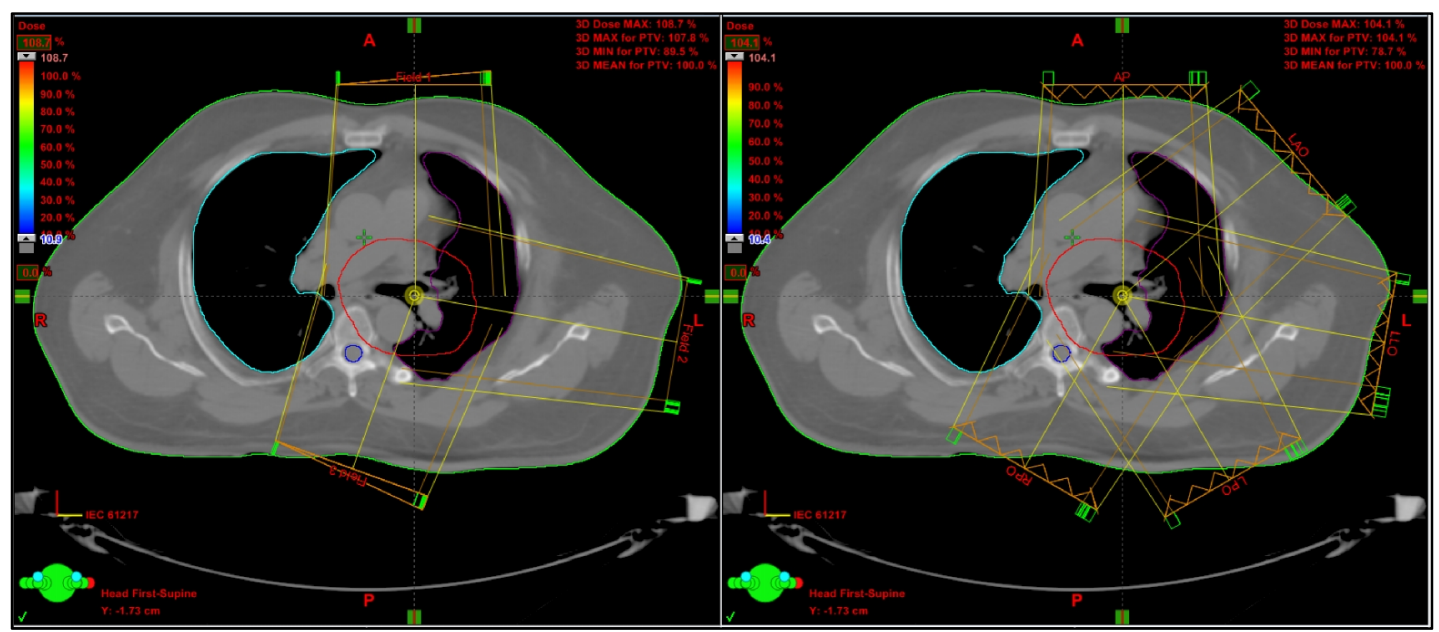

FIG. 1: Illustration of beam placement for a 3DCRT (left) and IMRT (right) plan for a lung case. It is clear that the beams are placed so as to avoid the contralateral lung. 


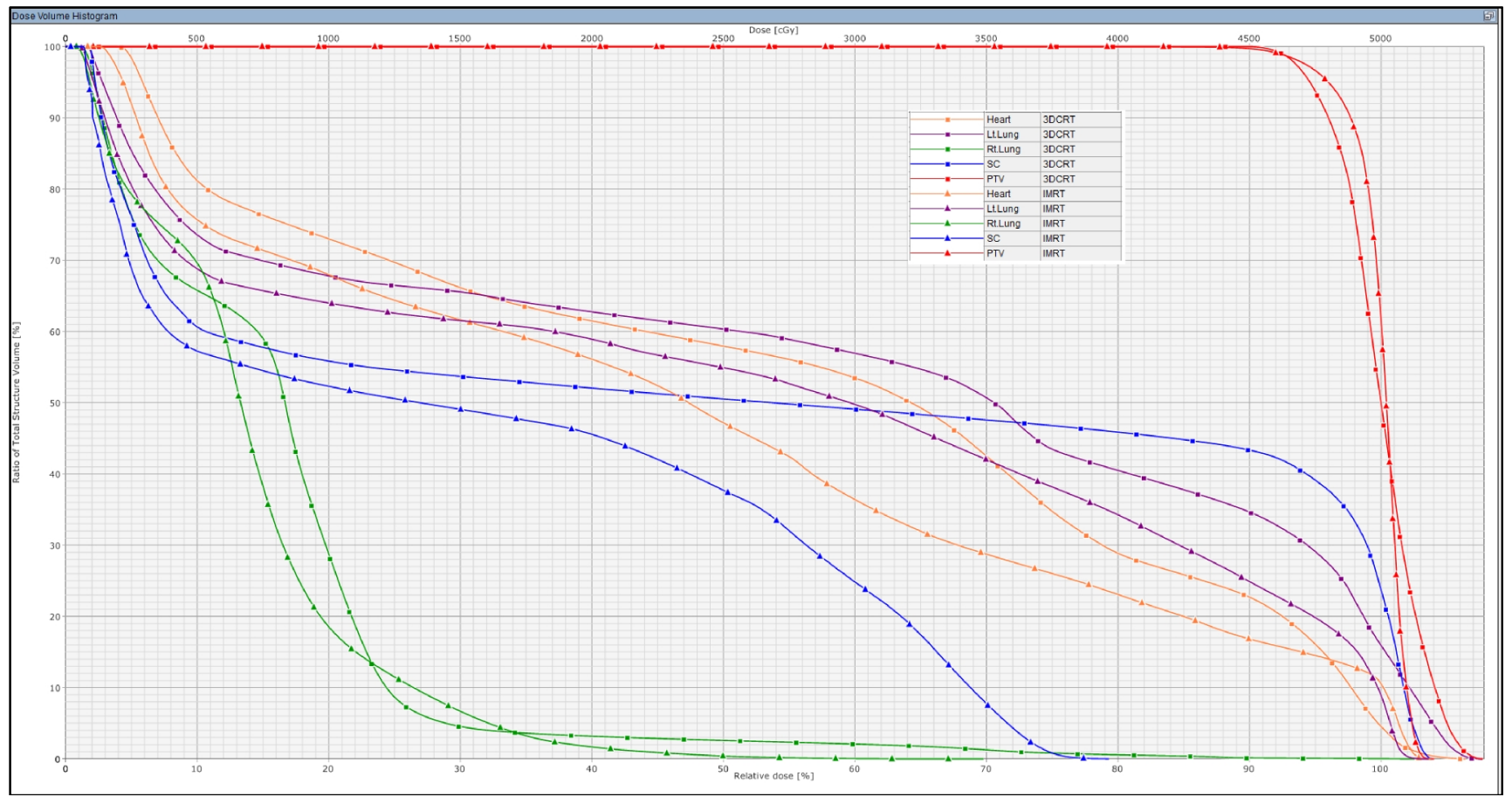

FIG. 2: Illustration of the dose-volume histogram obtained for a lung case for 3DCRT and IMRT with beam orientation as shown in the Figure 1.

The plans were analyzed on the basis of conformity index and dose to the critical structures. The conformity index is defined as the ratio of the volume of target volume receiving $95 \%$ of the prescribed dose to the total volume of the target volume. The plan which gives the better conformity while giving minimal dose to critical structures in both 3DCRT and IMRT is selected and taken for the first study. In this work, we have not compared the results between different energies because the low dose volume for higher energy beams is same as that of the lower energy beams. This is because of the fact that the reduction in dose due to buildup in higher energy fields is compensated by the higher exit dose and larger field boundary margins.

For the other analysis the plans which were formed with different number of beams were used.

In study 1, the best plan from 3DCRT and IMRT were compared by plotting graphs for $5 \mathrm{~Gy}, 10 \mathrm{~Gy}, 15 \mathrm{~Gy}$ and $20 \mathrm{~Gy}$ volumes. In study 2, 3DCRT plans alone with different number of beams were compared to analyze the $5 \mathrm{~Gy}, 10 \mathrm{~Gy}$, $15 \mathrm{~Gy}$ and $20 \mathrm{~Gy}$ volume regions as related to the increase in number of fields. In study 3, the above study was repeated for IMRT plans. In study 4, 3DCRT and IMRT plans with equal number of beams for example 5 field 3DCRT and 5 field IMRT were compared to see in which modality the low dose volume regions are higher.

\section{Results and Discussion}

The 5 Gy, 10 Gy, 15 Gy and 20 Gy dose volumes were analyzed in this project for all 3DCRT and IMRT plans.

\section{Study 1}

3DCRT plans were done for the delineated target volume and the best plan with highest dose coverage with minimal dose to adjacent normal tissues was selected for this study. For pelvic cases, box field plans seem to give better coverage while bilateral fields achieve the set goal for head and neck cases. For chest regions, similarly a plan which gives the better therapeutic ratio is selected. IMRT plans were also done for the same set of patients. The above mentioned criterion of higher conformity index with lesser dose to normal structures was selected.

In both, 3DCRT and IMRT plans 5 Gy, 10 Gy, 15 Gy, 20 Gy were measured and noted down. A graph was plotted with different dose levels (5 Gy, $10 \mathrm{~Gy}, 15 \mathrm{~Gy}$, and $20 \mathrm{~Gy}$ ) in the $\mathrm{X}$ - axis and volume in $\mathrm{Y}$-axis to analyze in which modality the low dose level regions were high. Few graphs are shown below. 


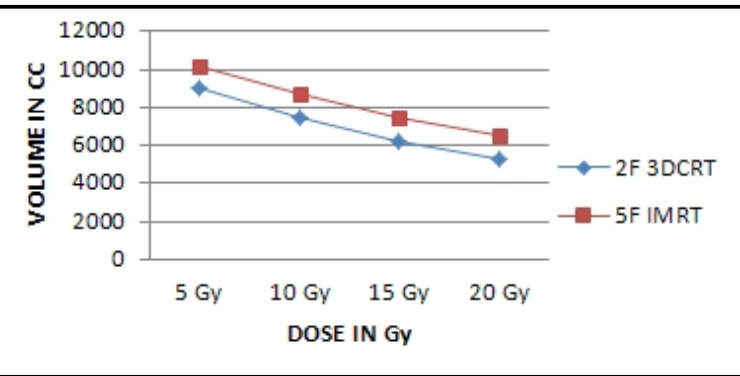

FIG. 3: Shows the illustration of the effect seen in head and neck regions. The blue line represents the 3DCRT while the red line represents the IMRT.

From the graph, it is clearly seen that the $5 \mathrm{~Gy}, 10 \mathrm{~Gy}, 15 \mathrm{~Gy}, 20 \mathrm{~Gy}$ volumes were high in IMRT while compared to that of 3DCRT.

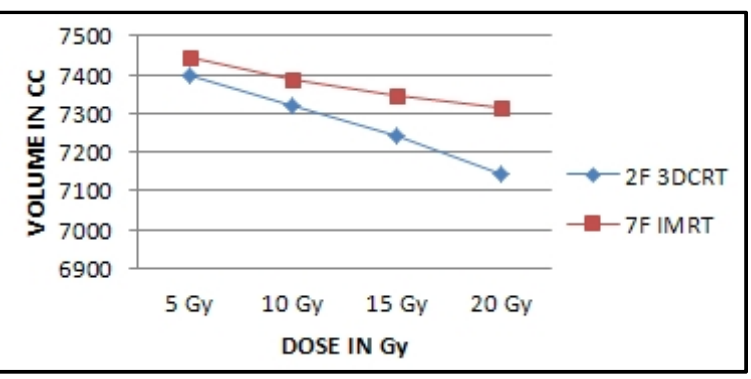

FIG. 4: Shows illustration of a head and neck case.

Clearly it is evident from the graph that for head and neck regions, the low dose level regions were higher in IMRT than in the 3DCRT Plans.

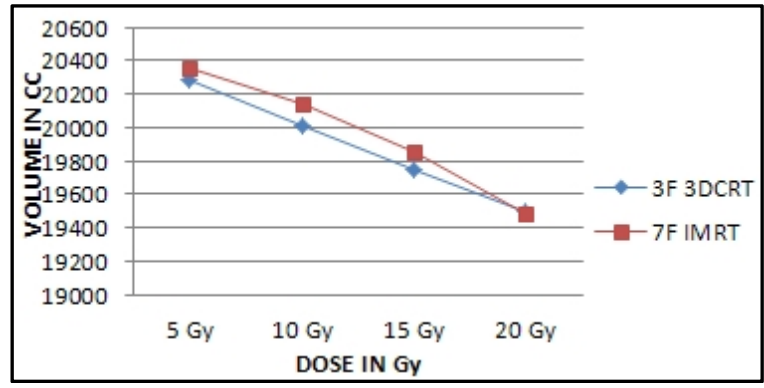

FIG. 5: Shows the illustration of a lung case. The blue line represents the 3DCRT plan with three fields and the red line represents the IMRT plan.

The effect observed in chest region is similar to that of the head and neck regions with slight variations.

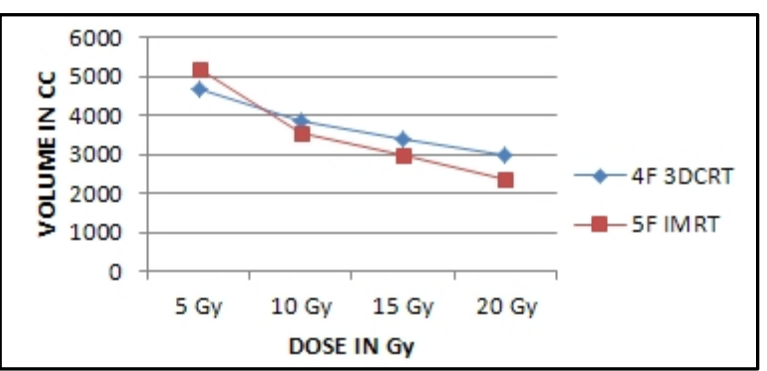

FIG. 6: Shows the illustration of a pelvic case. The blue line represents the 3DCRT Box Field Plan while the red line represents an IMRT Plan.
The 5Gy volume was high in IMRT while the others were lesser.

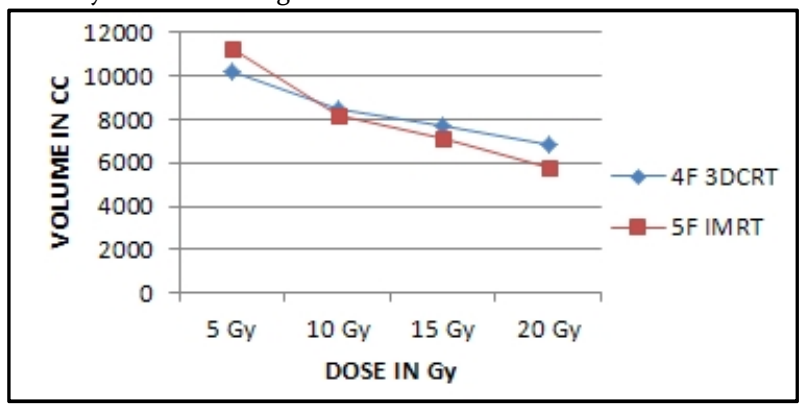

FIG. 7: Illustrates a pelvic case which is similar to previous graphs.

In pelvic cases a different effect was observed as compared to the effect in cases of head and neck and lung region. Only the 5 Gy volume regions was high in IMRT plans while $10 \mathrm{~Gy}, 15$ Gy and 20 Gy volumes were high in 3DCRT plans.

\section{Study 2}

In this study the low dose volume regions $5 \mathrm{~Gy}, 10 \mathrm{~Gy}, 15 \mathrm{~Gy}$, 20 Gy were analyzed for 3DCRT plans with different number of fields. When the number of beam portals is increased the area of irradiation is certainly increased. In this study an attempt was made to analyze the effect on low dose regions 5 Gy, 10 Gy, 15 Gy, 20 Gy as the number of beams increases.

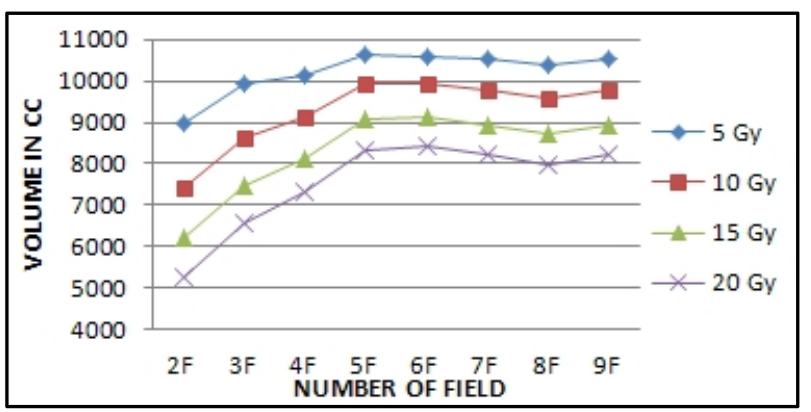

FIG. 8: Shows a head and neck case.

The low dose volume regions seem to increase and then saturate at five fields after which it remains almost the same.

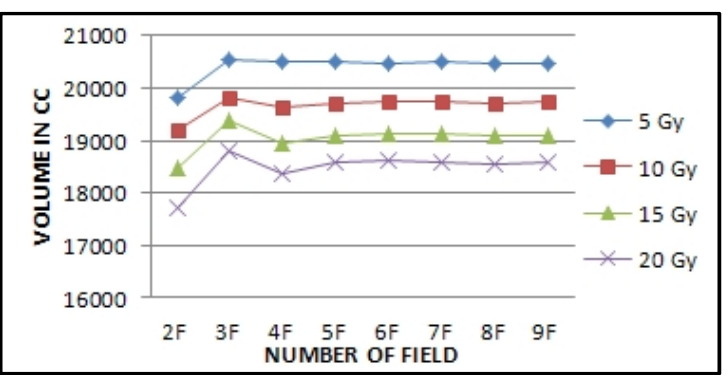

FIG. 9: Shows the illustration of a lung case where the low dose regions increases and then saturates.

The sudden dip in the graph was because when parallel opposing fields were used the low dose regions were less as the doses add up. 


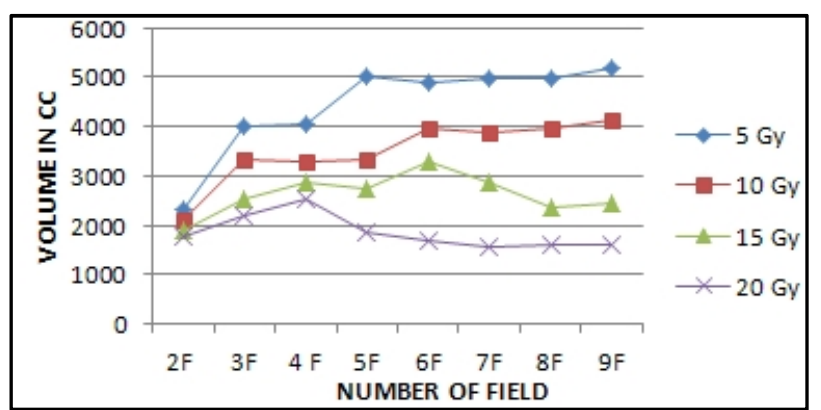

FIG. 10: Shows the illustration of a pelvic case, here the same effect as seen in lung case is observed.

The dips in graph were seen in plans with parallel opposing fields.

\section{Study 3}

In this study, the above analysis is repeated but for IMRT plans. IMRT plans with five to nine number of fields were done and graphs were plotted as before.

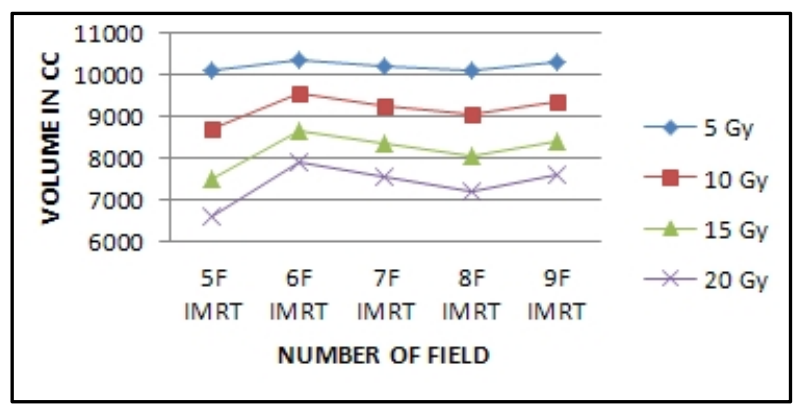

FIG. 11: Shows the illustration of a head and neck case.

As the number of fields increases the low dose volume regions increased and then remained almost the same.

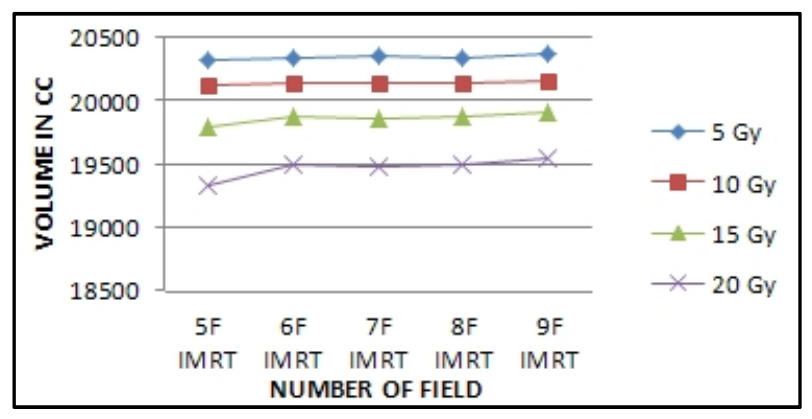

FIG. 12: This graph is drawn for a lung case.

This graph shows the same behavior as the previous graph.

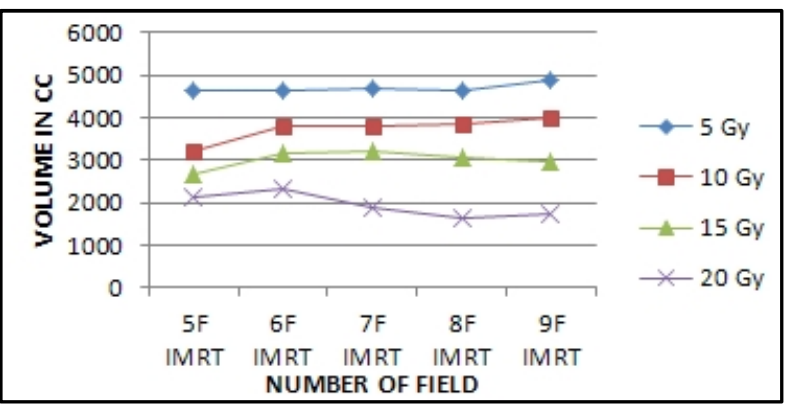

FIG. 13: This graph is an illustration of a pelvic case.

The same effect seen in the previous two graphs are observed in this graph also. So immaterial of whether it is head and neck or lung case or pelvic case as the number of fields increases there was a slight increase in the low dose volume regions and then remains almost the same.

\section{Study 4}

In this study, the above two studies are compared. With the same number of fields the low dose regions in 3DCRT and IMRT are compared to see in which modality the low dose regions are high.

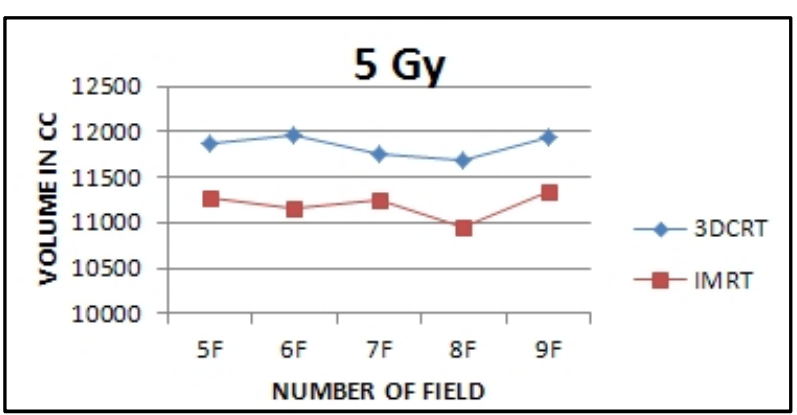

FIG. 14: Shows the $5 \mathrm{~Gy}$ volume regions for pelvic case in both 3DCRT and IMRT

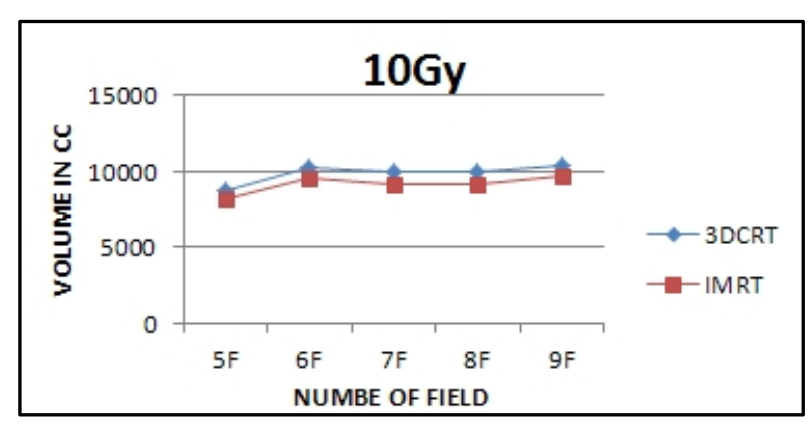

FIG. 15: Shows the 10 Gy volume regions for pelvic case in both 3DCRT and IMRT 


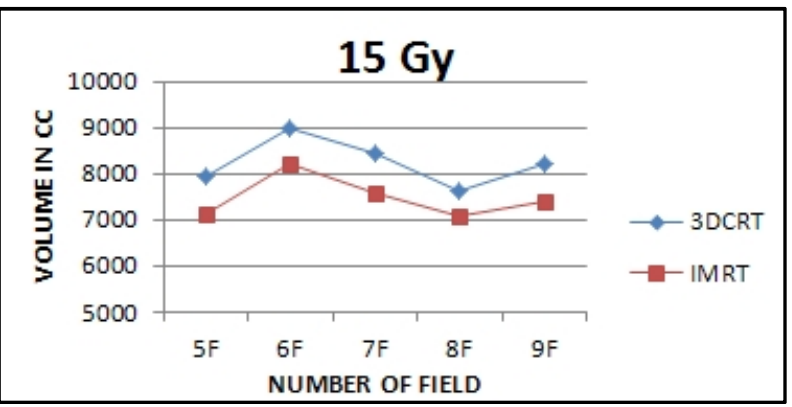

FIG. 16: Shows the 15 Gy volume regions for pelvic case in both 3DCRT and IMRT

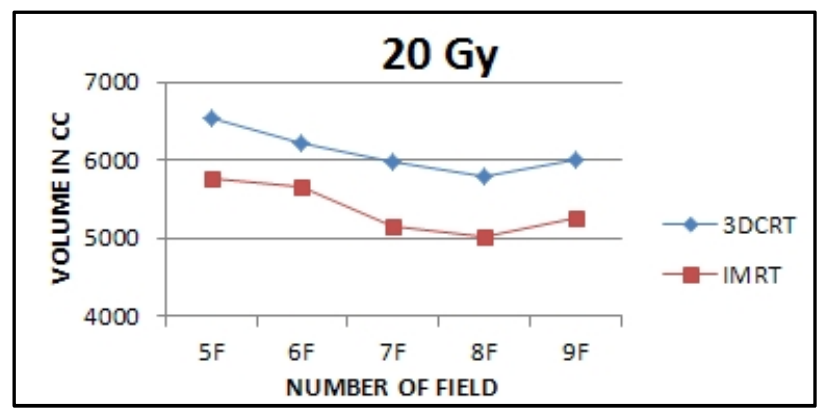

FIG. 17: Shows the 20 Gy volume regions for pelvic case in both 3DCRT and IMRT.
The above graphs were illustrative of one pelvic case. The 5 Gy volume regions were high in 3DCRT than in IMRT. While the other dose volume regions were also high in 3DCRT but the variation was considerably less. Only in 5 Gy volume regions the effect was highly pronounced.

The above behavior which was observed in pelvic case was seen in all head and neck and lung cases. It is clearly evident that the low dose volume regions are certainly higher in 3DCRT plans than the IMRT plans with the same number of fields.

The same study can be extended to higher modalities like dynamic arc treatments (VMAT) etc. Many studies are already going on persisting to this project. In VMAT the degree of freedom to deliver the desired dose to the target is higher as the gantry rotates around the target while simultaneously changing dose rate and field shapes. This causes many controversial views some proving that in spite of multiple beam angles the low dose volumes can be minimized by proper optimization. Still many research works are necessary to substantiate the low dose volume regions in dynamic treatment deliveries. ${ }^{13-17}$

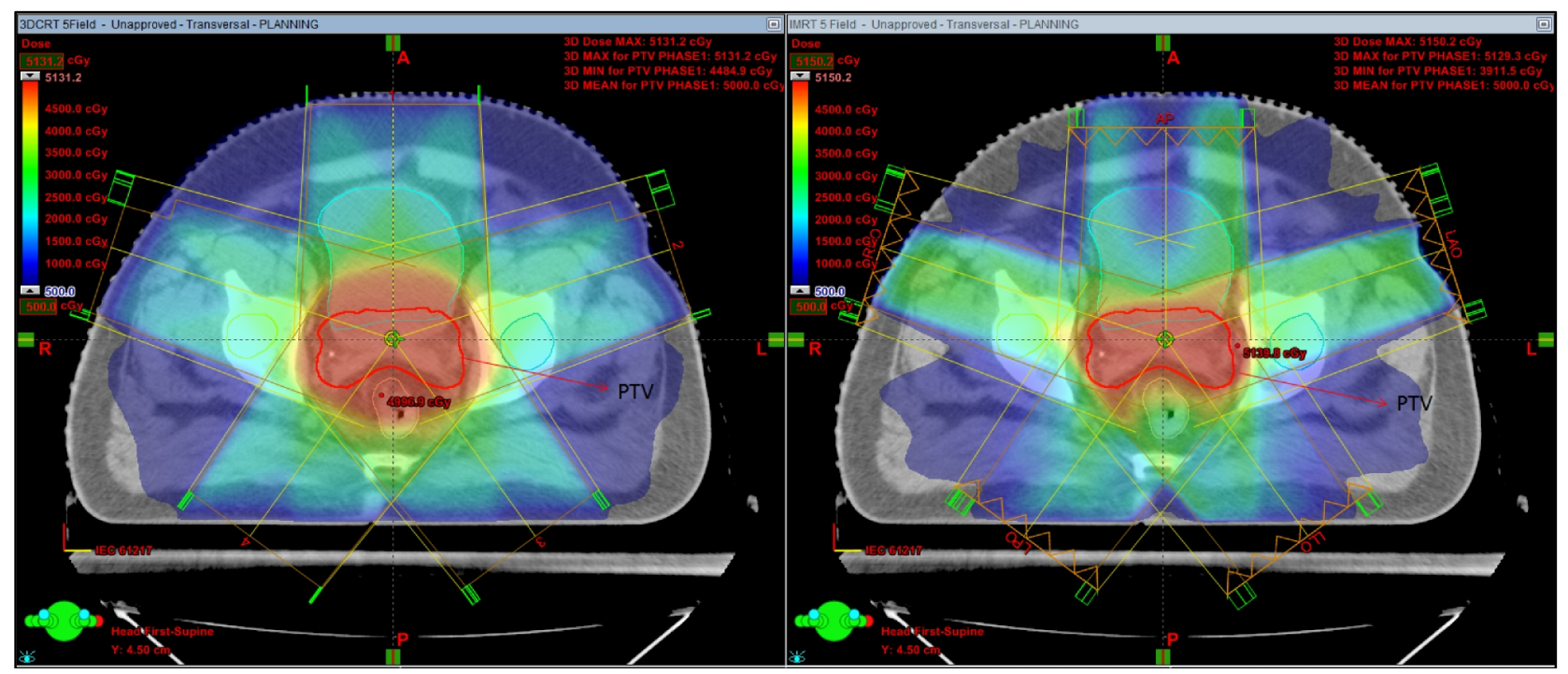

FIG. 18: The above figure is representative of the graphs obtained in this study. Left figure shows the 3DCRT dose distribution levels while the right represents that of the IMRT.

\section{Conclusion}

Low dose regions are very high risk regions in the radiotherapy treatment. The incidence of secondary cancer risk increases because of the unnecessary small doses. ${ }^{11}$ However, it cannot be avoided completely but careful planning is essential to minimize these low dose volume regions. With newer technologies the possibility to treat the target from different orientations are possible which could lead to higher regions of low dose levels. This study is aimed in analyzing these low dose volume regions.

From Study 1, it is clear that in IMRT the low dose volume regions are higher than that in 3DCRT. From study 2 and study 3 it is seen that immaterial of the modality as the number of fields increases the low dose volume regions increases and then saturates. From study 4 it is evident with the same number of fields the low dose regions are higher in 3DCRT than the IMRT plans. From these analysis, it is clear 
that in every modality depending on the fields the low dose regions could be higher. So bearing this in mind, planning should be done to achieve the best optimized plan.

Since we have Eclipse 10 version treatment planning system with $\mathrm{PBC}$ and AAA algorithm only, we have performed this study using AAA. Nowadays, advanced algorithm such as Acuros $\mathrm{XB}$ is available for dose calculations, and such advanced algorithm could predict more accurate dose than the algorithms of previous generations. ${ }^{18-21} \mathrm{We}$ would like to suggest the readers to investigate the impact of the advanced dose calculation algorithms on the low-dose volumes in the plans created by IMRT and 3DCRT.

\section{Conflict of interest}

The authors declare that they have no conflicts of interest. The authors alone are responsible for the content and writing of the paper.

\section{References}

1. Purdy JA. Advances in three-dimensional treatment planning and conformal dose delivery. Semin Oncol 1997; 24:655-71.

2. Aoyama H, Westerly DC, Mackie TR, et al. Integral radiation dose to normal structures with conformal external beam radiation. Int $J$ Radiat Oncol Biol Phys 2006; 64:962-7.

3. Hermanto U, Frija EK, Lii MJ, et al. Intensity-modulated radiotherapy (IMRT) and conventional three-dimensional conformal radiotherapy for high-grade gliomas: does IMRT increase the integral dose to normal brain? Int J Radiat Oncol Biol Phys 2007; 67:1135-44.

4. Purdy JA. Dose to normal tissues outside the radiation therapy patient's treated volume: a review of different radiation therapy techniques. Health Phys 2008; 95:666-76.

5. Yang $\mathrm{R}, \mathrm{Xu} \mathrm{S}$, Jiang $\mathrm{W}$, et al. Integral dose in three-dimensional conformal radiotherapy, intensity-modulated radiotherapy and helical Tomotherapy. Clin Oncol ( $R$ Coll Radiol) 2009; 21:706-12.

6. Hall EJ. Radiobiology for the Radiologist. Lippincott, Philadelphia, PA, 2000.

7. Steel GG. Basic Clinical Radiobiology. Arnold, London, 2002.

8. Vorwerk H, Wagner D, Seitz B, et al. Overestimation of low-dose radiation in intensity-modulated radiotherapy with sliding-window technique. Strahlenther Onkol 2009; 185:821-9.

9. Jang SY, Liu HH, Mohan R. Underestimation of low-dose radiation in treatment planning of inten- sity - modulated radiotherapy. Int J Radiat Oncol Biol Phys 2008; 71:1537-46.

10. Aoyama H, Westerly DC, Mackie TR, et al. Integral radiation dose to normal structures with conformal external beam radiation. Int I Radiat Oncol Biol Phys 2006; 64:962-7.

11. Klaus-Rüdiger Trott. Can we reduce the incidence of second primary malignancies occurring after radiotherapy? Radiotherapy and Oncology 2009; 91:1-3.

12. Susan $R$ Rheingold, MD, Alfred I Neugut, MD, $\mathrm{PhD}$, and Anna T Meadows, MD. Holland-Frei Cancer Medicine. $5^{\text {th }}$ edition. Section 40, Chapter 156: Secondary Cancers: Incidence, Risk Factors, and Management. Hamilton (ON): BC Decker; 2000.

13. Moorthy S, Sakr H, Hasan S, Samuel J, et al. Dosimetric study of SIB-IMRT versus SIB-3DCRT for breast cancer with breath-hold gated technique. Int J Cancer Ther Oncol 2013; 1:010110.

14. Pokharel S. Dosimetric impact of mixed-energy volumetric modulated arc therapy plans for high-risk prostate cancer. Int J Cancer Ther Oncol 2013; 1:01011.

15. Abbas AS, Moseley D, Kassam Z, et al. Volumetric-modulated arc therapy for the treatment of a large planning target volume in thoracic esophageal cancer. J Appl Clin Med Phys 2013; 14:4269.

16. Park J, Choi C, Ye S, Ha S. The dosimetric effect of mixed-energy IMRT plans for prostate cancer. $J$ Appl Clin Med Phy 2011; 12:3563.

17. Yin L, Wu H, Gong J, et al. Volumetric-modulated arc therapy vs. c-IMRT in esophageal cancer: a treatment planning comparison. World J Gastroenterol 2012; 18:5266 -75.

18. Rana S. Clinical dosimetric impact of Acuros $X B$ and analytical anisotropic algorithm (AAA) on real lung cancer treatment plans: review. Int J Cancer Ther Oncol 2014; 2:02019.

19. Lu L. Dose calculation algorithms in external beam photon radiation therapy. Int J Cancer Ther Oncol 2013; 1:01025.

20. Rana S, Rogers K, Pokharel S, Cheng C. Evaluation of Acuros XB algorithm based on RTOG 0813 dosimetric criteria for SBRT lung treatment with RapidArc. J Appl Clin Med Phys 2014; 15:4474.

21. Fogliata A, Nicolini G, Clivio A, et al. Critical appraisal of Acuros XB and Anisotropic Analytic Algorithm dose calculation in advanced non-small-cell lung cancer treatments. Int J Radiat Oncol Biol Phys 2012; 83:1587-95. 